\title{
On the Mechanism of H-Induced Curie-Point Relaxation in Fe-Based Glassy Alloys
}

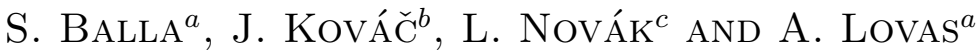

${ }^{a}$ Department of Vehicles Manufacturing and Repairing, BUTE

Bertalan L. 2, 1111 Budapest, Hungary

${ }^{b}$ Inst. of Exp. Physics, Slovak Academy of Sciences

Watsonova 47, 04001 Košice, Slovakia

${ }^{c}$ Department of Physics, Technical University of Košice

Park Komenského 2, 04200 Košice, Slovakia

\begin{abstract}
In Fe based glasses not solely the stress-sensitive magnetic properties, but also the amorphous Curie temperature $\left(T_{\mathrm{C}}^{\mathrm{am}}\right)$ can be altered by hydrogen absorption or due to the low temperature "heat treatments" in liquid $\mathrm{N}_{2}$ $\left(-196^{\circ} \mathrm{C}\right)$. The sign and magnitude of this $T_{\mathrm{C}}^{\mathrm{am}}$ shift is strongly compositional dependent, but it is also influenced by the thermal history of glasses (heat treatments). In this paper new experimental results are presented, which were obtained on FeCrB alloys, supporting the previous observations. An attempt of interpretation of the results is also made, which is based on the existence of the quenched-in phase reminiscences, being inherited from the liquid structure.
\end{abstract}

PACS numbers: 71.23.Cq, 71.55.Jv, 75.50.Kj, 81.40.Rs

\section{Introduction}

Irreversible and reversible Curie point $\left(T_{\mathrm{C}}^{\mathrm{am}}\right)$ relaxations are distinguished in the literature. The irreversible types are usually observed during the first period of isothermal heat treatments [1]. (This often appears as a significant increase related to the as-quenched state.) The corresponding structural change is believed to be a "topological short range ordering". In contrast, the reversible part of $T_{\mathrm{C}}^{\mathrm{am}}$ is associated with collective regrouping of atoms in the first neighbor environment (within short-range order scale). The change in ferromagnetic coupling is involved in both reordering types through the local change of atomic distances. It was reported $[2,3]$ that in Fe based glasses not solely the stress-sensitive magnetic properties, but also the amorphous Curie temperature $\left(T_{\mathrm{C}}^{\mathrm{am}}\right)$ can be altered by 
hydrogen absorption or due to the low temperature "heat treatments" in liquid $\mathrm{N} 2\left(-196^{\circ} \mathrm{C}\right)$. This $\mathrm{H}$-induced shift resembles "relaxation effect" as in many case appears as $T_{\mathrm{C}}^{\mathrm{am}}$ increase. In the present paper, some new results will be presented obtained in FeCrB glasses. The interpretation is based on the existence of the "frozen-in phase reminiscences" in the Fe-based glasses.

\section{Experimental}

The $10 \mathrm{~mm}$ wide samples were prepared by planar flow casting (PFC) method. (The wide ribbons were necessary for the H-saturation experiments.)

The details of electrolytic H-saturation and the $T_{\mathrm{c}}$ measurements are described in earlier papers $[2,4]$.

\section{Results and discussion}

In Figs. 1 and 2 the results of repeated $T_{\mathrm{C}}^{\mathrm{am}}$ measurements are shown, which were performed on as quenched and annealed samples, respectively (the measuring "run" in the figures refer to three subsequent $T_{\mathrm{c}}$ determination, which were performed on the same sample). The samples were subject to the three various treatments prior to the $T_{\mathrm{C}}^{\mathrm{am}}$ measurements: one sample was previously electrolytically charged (saturated) with $\mathrm{H}$ (sign as H-charged), the second sample was immersed into liquid $\mathrm{N}_{2}\left(-196^{\circ} \mathrm{C}\right)$ for $24 \mathrm{~h}$ (LN treated). Both of treatments were applied on the third sample (H-ch.+LN tr.). The procedures were identical for the heat-treated samples $\left(250^{\circ} \mathrm{C}\right.$ for $24 \mathrm{~h}$, see Fig. $\left.1 \mathrm{~b}\right)$.
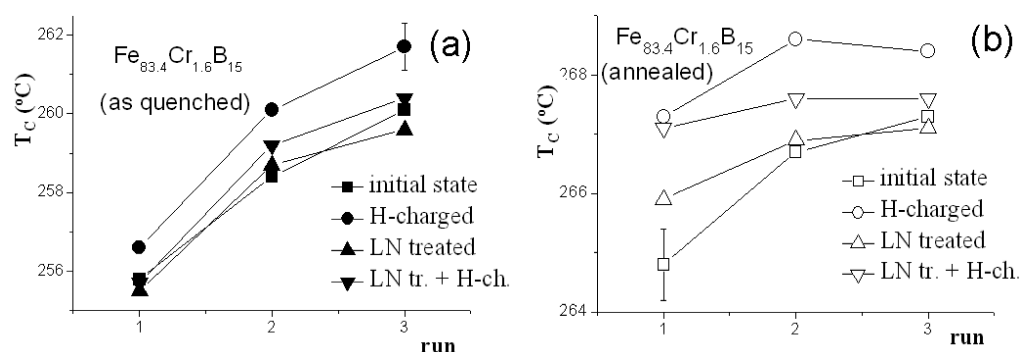

Fig. 1. The evolution of $T_{\mathrm{C}}^{\mathrm{am}}$ in $\mathrm{Fe}_{83.4} \mathrm{Cr}_{1.6} \mathrm{~B}_{15}$ samples being charged with $\mathrm{H}$ or "immersed into LN".

The $T_{\mathrm{C}}^{\mathrm{am}}$ is higher in the heat-treated samples as it is expected on the basis of previous (irreversible) structural relaxation. According to this irreversibility, the difference between as quenched and annealed samples is conserved during the subsequent measuring cycles. The sensitivity of the annealed samples to the same pre-treatments is higher for the composition of $\mathrm{Fe}_{83.4} \mathrm{Cr}_{1.6} \mathrm{~B}_{15}$. Both the $\mathrm{H}$-absorption and the LN treatment induced increase is conserved in the samples during the whole subsequent measuring cycles, indicating the stability of structural imprint caused by these treatments. 

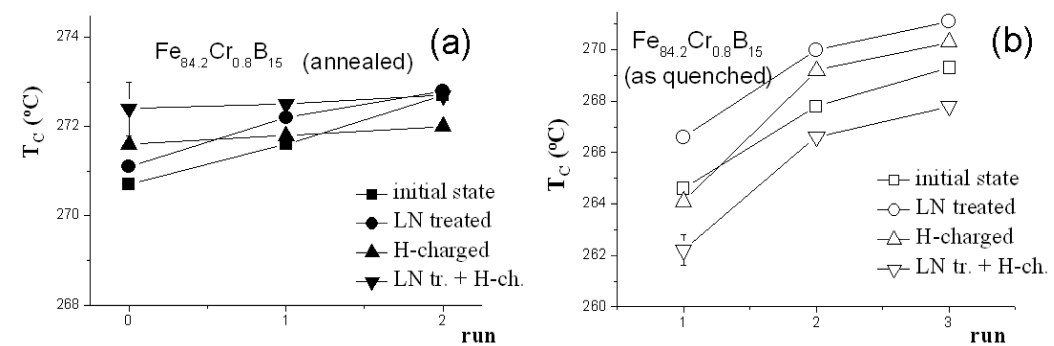

Fig. 2. The evolution of $T_{\mathrm{C}}^{\mathrm{am}}$ in as quenched and annealed $\mathrm{Fe}_{84.2} \mathrm{Cr}_{0.8} \mathrm{~B}_{15}$ samples after the same previous heat treatment as applied for the $\mathrm{Fe}_{83.4} \mathrm{Cr}_{1.6} \mathrm{~B}_{15}$ samples.

Analogically to the existence of independent diffusion and flow defects in the metallic glasses [5] one can suppose the preferential site-occupation of $\mathrm{H}$ atoms in individual, independent active centres, in which $\mathrm{H}$ entrap is governed by the local symmetry of atomic arrangements. As the solubility of $\mathrm{H}$ in the investigated systems does not exceed a few hundreds of ppm, a small fraction of atomic environments are responsible for the actual H-trapping only [6]. Such local symmetries are inherited from the frozen-in melt structure. Such preferential site-occupation (increased solubility) of $\mathrm{H}$-atoms is also observed in the crystalline pure $\mathrm{Fe}$ at the temperature of $\alpha-\gamma$ phase transition $\left(913^{\circ} \mathrm{C}\right)$ [7]. We assume that the "dense environments" are more susceptible to the H-entrap, like octahedral environments in the crystalline $\gamma$-Fe. Similar structural units would be active participants in the mechanism of H-solution in the Fe-based glasses [8]. The chemical interaction between the dissolved $\mathrm{H}$ atoms and the neighbouring (dominantly $\mathrm{Fe}$ ) atoms is weak, except the small, local volume expansion caused by the $\mathrm{H}$ cite-occupation. Due to this small expansion, the average atomic distances locally increases. In the spirit of Bethe-Slater conception the strength of ferromagnetic coupling is sensitive to the interatomic distances [9]. Therefore, one can expect that the local exchange interaction increases during the residence time of $\mathrm{H}$ atoms in these environments, resulting in the net increase in $T_{\mathrm{C}}^{\mathrm{am}}$. Phenomenologically, this increase resembles the $T_{\mathrm{C}}^{\mathrm{am}}$ shift caused by the relaxation heat treatments. The results in Figs. 1 and 2 hint to the concentration change of these centres when the sample is cooled to low temperature. The corresponding population change of $\mathrm{H}$-trapping sites can be understood on the basis of the phenomenological similarity existing between martensitic transformation (MT) and the glass transition [10]. The completion of MT often occurs only well below the room temperature in several steels [11]. Both transformations are non-diffusive and the completions are highly composition can and cooling rate dependent. If the temperature drops sufficiently below the room temperature, the MT transformation further proceeds and completes. Based on this similarity, one can assume that glass transformation is not fully completed when the $T_{\mathrm{g}}$ is reached during the liquid quench and additional (mainly shear-stress induced) atomic regroupping may occur in the glassy state when the sam- 
ple is cooled to sufficiently low temperatures such as LN $\left(-196^{\circ} \mathrm{C}\right)[12,13]$. More detailed description of this interpretation is going on.

\section{Conclusions}

The manifestations of H-absorption and "cryo treatment" are studied in the Curie temperature relaxation of Fe-based glasses:

- It was found that $T_{\mathrm{C}}^{\mathrm{am}}$ increases due to the electrolytic H-charging in the investigated alloys.

- Similar $T_{\mathrm{C}}^{\mathrm{am}}$ increase was detected in the same alloys after resting the samples at low temperature $\left(-196^{\circ} \mathrm{C}\right)$.

- The effects are interpreted by assuming the co-existence of independent, stressed and compressed (fcc and bcc-like) phase reminiscences.

\section{Acknowledgments}

This work was supported by the Hungarian Fund (OTKA) through grant No. T 046239 and Slovakian Funds VEGA 2/7193/27 and Cex NANOSMART.

\section{References}

[1] A. Inoue, H.S. Chen, T. Masumoto, S.A. Ajuria, Sci. Rep. Inst. Tohoku Univ., Ser. A 32, 116 (1985).

[2] L. Novák, A. Lovas, L.F. Kiss, J. Appl. Phys. 98, 043904 (2005).

[3] L. Novák, K. Bán, J. Kováč, A. Lovas, J. Magn. Magn. Mater. 304, 669 (2006).

[4] A. Stančáková, L. Kafková, L. Novák, E. Kisdi-Koszó, in: Proc. VI Scientific Conf., Technical University, Kosice 1992, p. 192.

[5] A. van den Beukel, Key Eng. Mat. 81-83, 3 (1993).

[6] K. Bán, A. Lovas, L. Novák, K. Csach, Czech. J. Phys. 54, Suppl. D, D137 (2004).

[7] J.D. Fast, Thermodynamics and Phase Relations, Interaction of Metals and Gases, Vol. 1, Philips Technical Library, Eindhoven 1965, p. 125.

[8] D. Srolovitz, T. Egami, V. Vitek, Phys. Rev. B 24, 6936 (1981).

[9] D. Jiles, Introduction to Magnetism and Magnetic Materials, St Edmundsburry Press, Bury St Edmunds, Suffolk 1998.

[10] A. Lovas, M. Vázquez, Acta Electrochemica Informatica 2, 86 (2002).

[11] Ö. Szabó, The Metallography of Iron and Steel Industry in Practice, Technical Press, Budapest 1968, p. 308.

[12] K. Bán, A. Lovas, J. Kováč, Czech. J. Phys. 54, Suppl. D, D141 (2004).

[13] W. Kauzmann, Chem. Rev. 43, 219 (1948). 